Págs. 139-156

\title{
Hacia una evaluación de la eficacia intercultural en el profesorado en formación
}

\author{
Towards an Evaluation of Intercultural Efficacy in Teacher Training
}

Tatiana Iñiguez-Berrozpe, Diana Valero, Ainhoa Flecha y Gisela Redondo-Sama ${ }^{1}$

\begin{abstract}
Resumen
En el presente artículo se realiza un análisis exploratorio del concepto de eficacia intercultural en relación con la profesión docente, aplicando la Multicultural Efficacy Scale de Guyton y Wesche (2005), (Escala de Eficacia Intercultural) recogiendo datos de n.=296 estudiantes de último año de Magisterio de Educación Infantil, Primaria y del Máster de Profesorado de Secundaria. Para su análisis se evalúa la incidencia de factores sociodemográficos en los índices de eficacia cultural, y la relación de dependencia entre ellos. Los resultados evidencian la aplicabilidad de la escala; que el profesorado en formación presenta índices elevados tanto en actitud como en eficacia intercultural, pero un escaso conocimiento sobre ello; se demuestra que las variables sociodemográficas inciden en los índices de eficacia intercultural; y cómo la actitud influye en la eficacia intercultural.
\end{abstract}

\section{Palabras clave}

Educación intercultural, educación multicultural, formación de profesores, interculturalidad.

\section{Abstract}

In this article an exploratory analysis of the concept of multicultural efficacy in relation to the teaching profession is carried out, applying the Multicultural Efficacy Scale by Guyton and Wesche (2005), collecting data of n. = 296 students of last year of Teaching of Early Education, Primary Education and the Master of Secondary Education Teachers. For its analysis, the incidence of sociodemographic factors in the multicultural efficacy indexes, and the dependency relationship among them, is evaluated. The results show the applicability of the scale; that the teachers in formation present high indexes as much in attitude as in intercultural effectiveness, but a little knowledge on it; it is demonstrated that the sociodemographic variables affect the multicultural efficacy indexes; and how attitude influences multicultural efficacy.

\section{Keywords}

Intercultural education, multicultural education, teacher training, interculturality.

\section{Cómo citar/Citation}

Iñiguez-Berrozpe, Tatiana; Valero, Diana; Flecha, Ainhoa y Redondo-Sama, Gisela (2021). Hacia una evaluación de la eficacia intercultural en el profesorado en formación. Revista de Sociología de la Educación-RASE, 14 (2), 139-156. http://dx.doi.org/10.7203/RASE.14.2.16634. 


\section{Introducción}

La multiculturalidad de las aulas es una realidad en las enseñanzas de Educación Infantil, Primaria y Secundaria de centros públicos en España donde el alumnado de origen extranjero representa un 10,5\%, un 10,5\% y un 10,6\%, respectivamente (Ministerio de Educación, Cultura y Deporte, 2018). Ello supone que maestros y profesores van a gestionar aulas en las que, al menos, uno/a de cada diez alumnos y alumnas pertenecen a otras culturas (Carrillo, Girbés-Peco, De Botton y Valls-Carol, 2017; Flecha y Soler, 2013). Las aulas se convierten, así, en espacios multiculturales que, si son gestionados de manera inclusiva y dialógica, pueden conducir a una interactividad igualitaria entre sus miembros y transformarse en espacios interculturales (Gómez Barreto, Gil Madrona y Martínez, 2017; Broc, 2018; García-Carrión, Molina-Luque y Roldán, 2017). Es por ello que numerosos autores han puesto el acento en la relevancia del profesorado como potencial artífice principal de esa gestión inclusiva de la diversidad de las aulas (Bennett, Niggle, y Stage, 1990; Cummins, 2001; Parekh, 2006). Para lograrlo, siguiendo a Guyton y Wesche (2005), existe una condición fundamental: que el profesorado sea eficaz en su docencia en un contexto multicultural, entendiendo que dicha eficacia está compuesta por un constructo que incluye conocimientos, comprensión, actitud y habilidad para gestionar la diversidad cultural en el aula (Bennett, Niggle y Stage, 1990; Herrera, 2018). Así, siguiendo a estos autores y a Guyton y Wesche (2005), en el presente estudio analizaremos esta eficacia intercultural como la suma del conocimiento y comprensión de esta diversidad cultural a través de la experiencia adquirida, la actitud personal hacia ella y la habilidad para gestionarla (o eficacia intercultural $)^{2}$.

La literatura también coincide que el medio fundamental para lograr dicha eficacia intercultural es la educación intercultural (Bennett, Niggle, y Stage, 1990: 244). Es por ello que en nuestra Investigación nos hemos centrado fundamentalmente en el profesorado en formación, de tal forma que analicemos dicha eficacia antes de que se incorporen a su actividad profesional. En este sentido, también debemos tener en cuenta que, al no haberse incorporado todavía a su actividad profesional, este profesorado en formación tenga todavía ciertas limitaciones en las competencias docentes adquiridas, como puede ser el caso de la eficacia intercultural. En general, este tipo de formación se ha ido incorporando a los planes de estudios de los Grados de Magisterio tanto de forma directa (como ejemplo tenemos la asignatura «Educación Intercultural» en la Universidad de Zaragoza), como transversal a lo largo de los 4 años de carrera. En el máster de profesorado, al ser tan solo de un año académico de duración ${ }^{3}$, es un tipo de contenido que se ve a menudo de manera indirecta.

Sin embargo, a pesar de que la gestión eficaz de la diversidad cultural en las aulas ha ido recibiendo un mayor interés por parte de la literatura científica, en habla hispana esta investigación se encuentra en un estado semi-embrionario, no habiéndose desarrollado apenas instrumentos que la permitan medir de modo fiable. En consecuencia, en la presente investigación proponemos como herramienta la traducción y adaptación al contexto español de la Multicultural Efficacy Scale (MES) de Guyton y Wesche (2005) [denominada Escala de Eficacia Intercultural —EEI — en nuestra adaptación] $]^{4}$, la cual también se ha validado en el presente trabajo. En este sentido, mediante la recogida de datos a través de este cuestionario adapta-

\footnotetext{
Los ítems de cada uno de los constructos se detallan en la Tabla I.

En algunas universidades es de dos cursos académicos.

4 El concepto anglosajón multicultural, traducido al español, se usa para describir una determinada situación en la que se refleja una pluralidad cultural existente en un espacio, independientemente de las relaciones que se den entre sus sujetos. Es por ello que, en esta investigación se ha decidido utilizar el término más apropiado en nuestro idioma de intercultural. Éste hace referencia a cómo son las relaciones, las interacciones que se dan y que se establecen entre las personas de las diferentes culturas en estos espacios de diversidad, más ajustado a lo que en inglés se entiende como multicultural (Romero, 2003).La interculturalidad implica una voluntad, una dirección hacia la cual queremos orientar las relaciones entre las culturas, siendo esta dirección la coexistencia y la convivencia.
} 
do, se han analizado las respuestas de n.=296 estudiantes de último año de Magisterio de Infantil y Primaria, y del Máster de Profesorado de Secundaria. Para medir el constructo antedicho se han construido tres índices: el Índice de Experiencia Intercultural (IEI), el Índice de Actitud Intercultural (IAI), y el Índice de Eficacia Intercultural (IEFI). Además del análisis descriptivo de estos índices, se ha llevado a cabo un estudio estadístico multivariante para mostrar sus relaciones de dependencia, así como la incidencia de las variables sociopersonales y educativas en la presencia de una mayor o menor puntuación en cada índice.

Los resultados demuestran que, en general, la experiencia intercultural es media o media-baja entre la mayoría de los/las participantes, si bien la actitud y la eficacia intercultural se encuentran en valores medio-altos. No obstante, el conocimiento o enfoque sobre la interculturalidad es superficial en más de la mitad de los/las participantes. Por otro lado, se constata la influencia de la actitud en la eficacia intercultural, así como la incidencia de variables como el sexo, la edad o el tipo de estudios cursados en estos índices. Finalmente, a nivel metodológico, se constata la validez y fiabilidad del instrumento empleado para explorar la eficacia intercultural entre el profesorado en formación, también en el contexto español.

\section{Revisión de la Literatura}

Tal y como especificábamos en la introducción, los flujos migratorios hacia Europa en los últimos 20 años han cambiado substancialmente la composición de las aulas (Gao y Mager, 2011). Si en el año 2000, en España, el alumnado inmigrante no superaba el 2\%, en el último curso con resultados detallados (2015-2016, Ministerio de Educación, Cultura y Deporte, 2018), supone casi un 11 \% en los centros públicos en los tres niveles educativos analizados en el presente artículo.

Este fenómeno plantea el reto de gestión de aulas multiculturales tanto a los propios centros como al profesorado (Howard y Aleman, 2008), fundamentalmente por las diferencias en cuanto al rendimiento académico de estudiantes nativos/as e inmigrantes. Sin ir más lejos, en la última edición de la prueba PISA (Programme for International Student Assessment) (Organización para la Cooperación y el Desarrollo Económico, OCDE, 2016a), dicha diferencia llegaba a los 50 puntos en nuestro país. Obviamente esta cuestión es multicausal, pero, en los últimos años, diversos autores y autoras han puesto el acento en la influencia de las actitudes y creencias del profesorado en el rendimiento del alumnado inmigrante (Atwater, Lance, Woodard y Johnson, 2013; Im y Martin, 2015; Kirch, Bargerhuff, Cowan y Wheatly, 2007). Estos/ as y otros/as autores/as han evidenciado en sus estudios que, si el profesorado carece de las competencias y recursos necesarios para gestionar la diversidad cultural en el aula, puede atribuir al alumnado de otras culturas habilidades cognitivas menores (Sacks y Watnick, 2006), priorizar las experiencias vitales del alumnado (Nieto, 1993), o tener bajas expectativas en cuanto a la participación e interés de sus familiares en su educación (Colombo, 2005), lo cual, según Park, Chu y Martin (2016) influye decisivamente en el rendimiento de este alumnado de otras culturas. Por el contrario, si los y las docentes poseen conocimientos y competencias para la gestión de la multiculturalidad, o, lo que es lo mismo, eficacia intercultural (Nadelson et al., 2012), ésta puede revertir positivamente en el rendimiento de los y las estudiantes inmigrantes y en la convivencia en el aula (Martin y Dagostino-Kalniz, 2015; Gómez Barreto y otros, 2017).

Por tanto, debido a la relevancia de este enfoque, un volumen emergente de investigaciones ha comenzado la tarea de contextualizar la eficacia del profesorado con un enfoque explícito en la diversidad, la enseñanza culturalmente receptiva y la interculturalidad, en un intento de aumentar los esfuerzos para preparar a los y las docentes culturalmente receptivos/as y eficazmente interculturales (Park, Chu y Mar- 
tin, 2016). Si el concepto tradicional de eficacia, atribuido a Bandura (1978: 3) son «las capacidades de uno/a mismo/a para organizar y ejecutar los cursos de acción requeridos para producir logros dados», la eficacia intercultural del profesorado se definiría como el «enfoque democrático de enseñanza-aprendizaje que busca fomentar el pluralismo cultural dentro de sociedades culturalmente diversas» (Bennett, Niggle, y Stage, 1990: 244), también definida por la literatura como «educación intercultural» (Guyton y Wesche, 2005). Según Bennett, Niggle, y Stage (1990), las dimensiones de ésta serían el conocimiento de esta diversidad cultural, su comprensión, la actitud personal hacia ella y la habilidad para gestionarla (o eficacia).

Teniendo en cuenta estas dimensiones, Guyton y Wesche desarrollaron y validaron en 2005 su MES para profesorado o profesorado en formación, argumentando su enfoque inclusivo, ya que las escalas anteriores solo incluían una parte de ellas. Ejemplo de dichas escalas previas son: el Inventario de Identificación de Competencia Intercultural (Campbell y Farrell, 1985), la Encuesta de Opinión Intercultural (Ohio State University, 1988), el Inventario de Atención a la Diversidad Cultural Hadaway (Larke, 1990), en el que se miden habilidades y actitudes, la Encuesta de Diversidad Social (Anders,Martin, y Yarbrough, 1990) para mensurar experiencias y actitudes interculturales o la Autoevaluación de Educación Intercultural (Cooper, Beare y Thorman, 1990), que mide el conocimiento sobre las prácticas de enseñanza intercultural en el profesorado y si éste se siente cómodo con dichas prácticas.

Así, en un primer estudio piloto, Guyton y Wesche (2005) recogieron las respuestas de 665 estudiantes de magisterio de pregrado y postgrado en Estados Unidos, en una primera versión de la escala de 160 ítems medidos en escala Likert 1-4, que tenía en cuenta las cuatro dimensiones de interculturalidad antedicha. Tras un análisis factorial, los autores depuraron el cuestionario dejándolo en 80 elementos. Tras una segunda prueba, se confeccionó el MES final de 35 ítems: 7 ítems de experiencia, 7 ítems de actitud, 20 ítems de eficacia, con un ítem adicional pidiendo a los participantes que identifiquen sus principales enfoques o creencias sobre la enseñanza en entornos educativos multiculturales. En cuanto a este último punto, los autores se refieren a los enfoques del profesorado para la educación intercultural descritos por Sleeter y Grant (1987): a) La visión desde la tolerancia a otras culturas en el aula; b) la visión asimilacionista, por la cual se aboga por que el alumnado diverso sea ayudado a asimilar la cultura de acogida; c) la visión desde la identidad singular y valiosa de cada cultura; d) el llamado «enfoque intercultural», que enfatiza los beneficios del pluralismo cultural para la sociedad e intenta reducir los prejuicios basados en cualquier tipo de diversidad; e) la visión intercultural desde el apoyo social, que toma todas las ideas de los enfoques anteriores pero aboga por motivar a los y las estudiantes a tomar medidas apropiadas y efectivas para corregir las injusticias sociales. Este último enfoque sería el que más se aproxima a la idea de eficacia intercultural del profesorado que señalan los/las autores/as y hemos tomado en el presente estudio.

En cuanto a los resultados del estudio, Guyton y Wesche (2005) reportaron que la sección de experiencia intercultural no servía para mensurar la eficacia, pero era relevante como factor determinante hacia una mayor o menor actitud y eficacia interculturales. En cuanto a la actitud, las puntuaciones eran, en general, elevadas, al obtenerse un 4 de mediana, si bien la eficacia intercultural marcaba un 3 de mediana. Los resultados sobre los enfoques mostraban que el «d) enfoque intercultural» era el que obtenía un mayor porcentaje (41,9\%), si bien la e) la visión intercultural desde el apoyo social, tan sólo era marcada por el 8,1\% de los y las participantes. No obstante, el resultado principal fue la validación del MES como un medio para diagnosticar al profesorado o profesorado en formación en términos de habilidades, entendimiento, y actitud como un constructo de la eficacia intercultural en el aula. 
Posteriormente, otros estudios, fundamentalmente en Estados Unidos, han empleado la MES (o adaptaciones de la misma, como en Silverman, 2008). Nadelson (2012), en un estudio con alumnado de Magisterio, mostró resultados similares a los de Guyton y Wesche (2005), es decir, puntuaciones en torno al 3 en eficacia intercultural, pero indicaban que no existían relaciones significativas en cuanto a las características personales y estas puntuaciones. Silverman (2015), empleando la MES en profesorado de Educación Primaria que ya estaba ejerciendo, obtuvo también puntuaciones medias en eficacia intercultural, pero una importante relación entre la experiencia intercultural previa y una mayor eficacia intercultural en el aula, resultados similares a los obtenidos por Seak-Zoon (2015) en Corea. Mansuri (2017), centrándose en alumnado de Magisterio tanto de universidades públicas como privadas, reporta como resultado más significativo una mayor puntuación en eficacia intercultural en el estudiantado del primer tipo de universidades. Todos/as ellos/as, al igual que Guyton y Wesche indicaron la necesidad de fomentar la formación en eficacia intercultural en el profesorado tanto en la formación inicial como en la continua. De hecho, Mitchell (2009), en un estudio realizado en un taller de educación intercultural con profesorado de Primaria, demostró como los niveles de la escala MES mejoraban significativamente, tanto en actitud como en eficacia, tras la formación en este ámbito.

En España, hasta el momento no se ha empleado la MES para medir la eficacia intercultural del profesorado, pero en fechas recientes una serie de estudios han analizado la eficacia intercultural del profesorado (Díez Gutiérrez, 2014) y del profesorado en formación (Gil-Madrona, Gómez-Barreto, y González-Víllora, 2016; Gómez Barreto, Gil Madrona, y Martínez, 2017), además de la relevancia de la formación para incrementarla (Aguaded, De la Rubia y González, 2013; Aguado Odina, Gil Jaurena y Mata Benito, 2008; Goenechea, 2008; Jordán, 2007). Es sorprendente que en estos y otros estudios se constaten resultados similares. Esto es, la existencia de la creencia, tanto entre el estudiantado de Magisterio como entre el profesorado en activo, de poseer actitudes y eficacia interculturales, pero cierto desconocimiento en cuanto a su práctica en el aula, así como la constatación de la necesidad de incrementar las actividades formativas para mejorar la competencia intercultural de este colectivo.

\section{Metodología}

\subsection{Instrumento}

Como se ha especificado en la revisión de la literatura, la escala empleada para medir la educación intercultural entre el profesorado en formación de distintos niveles de escolarización fue el cuestionario diseñado por Guyton y Wesche (2005), MES, que tiene en cuenta la experiencia, actitudes y eficacia intercultural de los participantes encuestados y permite arrojar luz sobre la complejidad de medir la educación multicultural del futuro profesorado (2005: 21).

El instrumento traducido al español, y denominado EEI (ver Anexo I) cuenta con una primera sección de preguntas sociopersonales y educativas: edad, procedencia, estudios cursados, institución, etnia, religión, país de nacimiento del padre y la madre, nivel educativo terminado del padre y la madre, y estatus socioeconómico cuando el participante era un niño/a y en la actualidad. En la adaptación del cuestionario al español se modificaron algunas preguntas tras el pre-test realizado. Se eliminó la pregunta de orientación sexual, y la relativa al grado de discapacidad, por no ser considerados como relevantes en el pre-test para los objetivos de nuestra investigación. Sin embargo, se añadió la pregunta de país de procedencia del padre y la madre, debido a que se consideró que algunos de los y las participantes podrían ser inmigrantes de segunda generación, dada la estructura sociodemográfica de nuestro país. 
Seguidamente, la escala de eficacia intercultural propiamente dicha cuenta con 35 ítems, con subescalas de experiencia intercultural ( 7 ítems), actitud hacia la interculturalidad en el aula (7 ítems) y eficacia intercultural en la educación (20 ítems). En las 3 subescalas las puntuaciones pueden ir en un rango de 1 a 4, con las siguientes especificidades:

- Subescala de Experiencia: 1-Nunca; 2-Con muy poca frecuencia; 3-A veces; 4-Frecuentemente.

- Subescala de Actitud: 1-Totalmente en desacuerdo; 2-Más o menos en desacuerdo; 3-Más o menos de acuerdo; 4-Totalmente de acuerdo.

- Subescala de Eficacia: 1-Creo que no podría hacer esto muy bien; 2-Podría hacerlo, pero sería difícil para mí; 3- Podría hacerlo razonablemente, si tengo tiempo para prepararlo; 4-Estoy bastante seguro/a de que hacer esto sería fácil para mí.

Una pregunta adicional clasifica a los y las participantes según lo que entienden como el principal objetivo de la enseñanza multicultural. A esta sección los autores la denominan «enfoque intercultural», y cada una de las posibles respuestas se corresponde con una de las aproximaciones a la educación intercultural, especificadas en la sección anterior.

El primer paso para su utilización fue traducirla al español, y verificar su comprensión mediante un comité de expertos en Sociología y Psicología, dentro del área de Educación de nuestras universidades. Posteriormente, tras la recogida de datos, contando con $n .=296$ participantes, se procedió a analizar la fiabilidad interna de la escala traducida, resultando un valor de ,86 en el coeficiente del alpha de Cronbach para los 35 ítems (,89 en el original en inglés de Guyton y Wesche). Los coeficientes de las subescalas fueron de ,71 para la sección de experiencia intercultural (7 ítems), ,57 para las actitudes hacia la interculturalidad ( 7 ítems), y ,91 para la eficacia intercultural (20 ítems), el valor de alpha en la escala original para estas subescalas fue de ,78; ,72; y ,93 respectivamente. Seguidamente se analizó la correlación entre los ítems de cada subescala y la puntuación total de la misma, siendo en todos los casos de las tres subescalas $r>$,3. Por lo tanto, se demostró la fiabilidad de la escala de eficacia intercultural adaptada a nuestro contexto.

Finalmente, para analizar si, tras su traducción al español, se mantenía la coherencia de la estructura interna de la escala, se llevó a cabo un análisis factorial exploratorio mediante la metodología PCA (Principal Components Analysis). Tras el análisis, la inspección de la matriz de correlación reveló la presencia de muchos coeficientes $r>$,3, el valor de Kaiser-Meyer-Oklin fue de ,90, que excede el valor recomendado de ,60 (Kaiser, 1970; 1974) y la prueba de esfericidad de Barlett (Bartlett, 1954) alcanzó significación estadística $(p=0,00)$, apoyando la factorabilidad de la matriz de correlación. A continuación, el PCA reveló la presencia de siete componentes con valores propios superiores a 1. Una inspección del gráfico de sedimentación reveló una clara ruptura después del tercer componente, por lo que se decidió retener la solución de tres componentes. Para ayudar en la interpretación de estos tres componentes, se realizó la rotación Varimax. La solución rotada reveló que estos tres componentes explicaban un total del $44,0 \%$ de la varianza $(27,8 \%, 8,4 \%$ y $7,9 \%$ respectivamente). El resultado muestra que los tres componentes se corresponden a cada una de las tres subescalas o factores subyacentes, agrupando sus ítems correspondientes. Esto es, el primer componente agrupa a todos los ítems relacionados con la eficacia intercultural (items 15-34 del cuestionario), el segundo a los relacionados con las actitudes hacia la interculturalidad (ítems 8-14), y el tercero a la experiencia (ítems 1-7), quedando demostrada así la adecuación de los ítems para medir cada uno de estos tres factores subyacentes. 
Tabla I. Matriz de componente rotado del análisis factorial exploratorio

\begin{tabular}{|c|c|c|c|}
\hline & \multicolumn{3}{|c|}{ COMPONENTE } \\
\hline & 1 & 2 & 3 \\
\hline 21. Puedo presentar la diversidad de grupos en nuestra sociedad de una manera que construya el respeto mutuo. &, 765 & & \\
\hline 26. Puedo ayudar a los estudiantes a resolver situaciones problemáticas causadas por actitudes estereotipadas y/o prejuiciosas. &, 758 & & \\
\hline 34. Puedo involucrar a los estudiantes en la toma de decisiones y aclarar sus valores con respecto a los asuntos multiculturales. &, 754 & & \\
\hline 29. Puedo identificar soluciones a los problemas que puedan surgir como resultado de la diversidad. & ,746 & & \\
\hline 23. Puedo proporcionar actividades de enseñanza-aprendizaje mostrando cómo los prejuicios afectan a los individuos. & 743 & & \\
\hline 30. Puedo identificar los agentes sociales que influyen en las oportunidades de las personas diversas. & 731 & & \\
\hline 32. Puedo ayudar a los estudiantes a asumir la perspectiva de grupos étnicos y culturales diferentes a los suyos. &, 724 & & \\
\hline 24. Puedo planificar actividades para reducir los prejuicios hacia grupos diversos. & ,703 & & \\
\hline 16. Puedo adaptar los métodos de enseñanza-aprendizaje para satisfacer las necesidades de los estudiantes de grupos diversos. &, 693 & & \\
\hline 15. Puedo proporcionar actividades de enseñanza-aprendizaje para ayudar a los estudiantes a desarrollar estrategias de convivencia y resolución de conflictos entre culturas. & 689 & & \\
\hline 20. Puedo ayudar a los estudiantes a valorar sus propios prejuicios. & 687 & & \\
\hline 22. Puedo desarrollar actividades que aumenten la autoconfianza de los estudiantes diversos. & 665 & & \\
\hline 27. Puedo conseguir que los estudiantes de grupos diversos trabajen juntos. & ,665 & & \\
\hline 33. Puedo ayudar a los estudiantes a ver la historia y los acontecimientos actuales desde perspectivas diversas. & 663 & & \\
\hline 28. Puedo identificar prácticas escolares que pueden herir a los estudiantes diversos. & 654 & & \\
\hline 31. Puedo identificar las maneras en las que los grupos diversos contribuyen a nuestra sociedad plural. & 639 & & \\
\hline 17. Puedo desarrollar materiales de enseñanza-aprendizaje apropiados para un aula multicultural. & 617 & & \\
\hline 19. Puedo analizar materiales de enseñanza-aprendizaie para discernir posibles contenidos estereotípicos y/o prejuiciosos. & 607 & & \\
\hline 25. Puedo identificar sesgos culturales en los libros de texto usados en la escuela. &, 562 & & \\
\hline 18. Puedo desarrollar métodos de enseñanza-aprendizaie que desmitifiquen acerca de los grupos diversos. &, 554 & & \\
\hline 14. La biblioteca del colegio debería reflejar todas las diferencias raciales y culturales del aula. & & ,730 & \\
\hline 12. Es esencial incluir las perspectivas de grupos diversos cuando se enseñan contenidos sobre la historia europea. & & ,688 & \\
\hline 13. El currículo y los libros de texto deberían incluir las contribuciones de la mayoría, o todos, los grupos culturales de nuestra sociedad. & &, 641 & \\
\hline 8. Los profesores deberían adaptar los contenidos en clase para reflejar las diferentes culturas representadas en el aula. & & 612 & \\
\hline 9. Los/as profesores/as deberían proporcionar oportunidades al alumnado para compartir sus diferencias culturales en la comida, la forma de vestir, su vida familiar o sus creencias. & & ,610 & \\
\hline 11. Los chicos y chicas deberían ser enseñados por sus profesores/as acerca de su propio origen étnico y cultural. & &, 414 & \\
\hline 10. Discutir las tradiciones éticas y las creencias en la escuela lleva a discusiones y desunión entre alumnado de diferentes culturas. & & ,310 & \\
\hline 2. Fui a la escuela con personas diversas durante mi adolescencia. & & & ,758 \\
\hline 1. Cuando era niño/a jugaba con personas distintas a mí. & & & ,705 \\
\hline 7. Cuando era adolescente estaba en el mismo equipo o club con estudiantes diversos/as. & & & ,672 \\
\hline 3. En el barrio donde viví cuando era niño/a había diversidad de personas. & & & 641 \\
\hline 4. En el pasado elegi leer libros sobre personas distintas a mí. & & & , 476 \\
\hline 6. En el pasado elegi ver peliculas y programas de televisión sobre personas distintas a mí. & & &, 457 \\
\hline 5. Una persona diversa era uno de mis modelos a seguir cuando era niño/a. & & & ,406 \\
\hline
\end{tabular}

Método de extracción: análisis de componentes principales.

Método de rotación: Varimax con normalización Kaiser.

La rotación ha convergido en 5 iteraciones.

Componente 1: eficacia intercultural; Componente 2: actitudes hacia la interculturalidad; Componente 3: experiencia intercultural.

\subsection{Muestra}

Para llevar a cabo el estudio, una vez pre-testado el cuestionario, se distribuyó el mismo de forma online, empleando la plataforma Google Drive, a diversas universidades españolas y en los grados de Magisterio de Educación Infantil ( $4^{\circ}$ curso), Magisterio de Educación Primaria (4ºurso) y Máster en 
Profesorado de Educación Secundaria (curso único), mediante la técnica de muestreo no probabilístico por conveniencia, empleando los contactos que teníamos en diversas universidades. La respuesta final fue de $n .=296$ cuestionarios válidos y completados en su totalidad, con un error muestral del $5,7 \% 5$. Esta muestra total se divide en un 36,1\% de estudiantes de Magisterio Infantil, un 14,5\% de Magisterio de Primaria, y un 49,3\% del Máster de Secundaria (tabla II).

El perfil de los y las participantes también se muestra en la tabla 2. En ella apreciamos un mayoritario número de estudiantes entre 20 y 25 años $(65,5 \%)$, mujeres $(81,4 \%)$, de religión cristiana $(57,4 \%)$, desempleados/as (58,4\%), y de nivel socioeconómico medio (36,1\%). En cuanto otros datos relacionados con sus familias, en la mayoría de los casos tanto el padre como la madre habían completado estudios de Secundaria o Formación Profesional (47,6\% y 45,6\% respectivamente), y decían haber tenido un estatus socioeconómico medio cuando eran niños $(34,5 \%)$.

Al tratarse de un análisis exploratorio, la muestra adolece de representatividad suficiente, dado que hemos tenido en cuenta un universo amplio de estudiantes. Si bien considerábamos que, en este punto del análisis (explorar la viabilidad de la escala para medir la eficacia intercultural en el profesorado en formación en España), debíamos tener en cuenta las tres especialidades (Infantil, Primaria y Secundaria) y evaluar la fiabilidad de esta propuesta de medición, así como explorar estas cuestiones relacionadas con la experiencia, la actitud y la eficacia interculturales en el futuro profesorado.

Tabla II. Distribución de la muestra por características sociodemográficas y educativas

\begin{tabular}{|c|c|c|c|}
\hline & INDICADORES & FRECUENCIA & PORCENTAJE \\
\hline \multirow{4}{*}{ Edad } & $20-25$ & 194 & 65,5 \\
\hline & $26-31$ & 66 & 22,3 \\
\hline & $32-37$ & 23 & 7,8 \\
\hline & $38-44$ & 13 & 4,4 \\
\hline \multirow{2}{*}{ Género } & Hombre & 55 & 18,6 \\
\hline & Mujer & 241 & 81,4 \\
\hline \multirow{3}{*}{ Estudios cursados } & Magisterio de Infantil & 107 & 36,1 \\
\hline & Magisterio de Primaria & 43 & 14,5 \\
\hline & Máster en Profesorado de Secundaria & 146 & 49,3 \\
\hline \multirow{3}{*}{ Religión } & Cristiana & 170 & 57,4 \\
\hline & Ninguna & 125 & 42,2 \\
\hline & Perdidos & 1 & 0,3 \\
\hline \multirow{5}{*}{ Nivel de estudios terminados por el padre } & Ninguno & 12 & 4,1 \\
\hline & Primaria & 60 & 20,3 \\
\hline & Secundaria/Formación Profesional & 141 & 47,6 \\
\hline & Diplomatura/Licenciatura universitaria & 70 & 23,6 \\
\hline & Doctorado & 7 & 2,4 \\
\hline \multirow{6}{*}{ Nivel de estudios terminados por la madre } & Ninguno & 7 & 2,4 \\
\hline & Primaria & 64 & 21,6 \\
\hline & Secundaria/Formación Profesional & 135 & 45,6 \\
\hline & Diplomatura/Licenciatura universitaria & 80 & 27 \\
\hline & Doctorado & 4 & 1,4 \\
\hline & NS/NC & 6 & 2 \\
\hline
\end{tabular}

5 Teniendo en cuenta un universo de 146977 estudiantes, que cursaron algunos de los niveles recogidos en el presente estudio (teniendo en cuenta el alumnado total de Magisterio de Educación Infantil y Primaria, sin diferenciar por curso, y el Máster de Secundaria, según datos del Ministerio de Educación y Cultura, 2017), considerando $\mathrm{p}=\mathrm{q}=0,5$, y un nivel de confianza del $95 \%$. 


\begin{tabular}{|c|c|c|c|}
\hline & INDICADORES & FRECUENCIA & PORCENTAJE \\
\hline \multirow{5}{*}{ Situación laboral actual del encuestado/a } & Desempleado & 173 & 58,4 \\
\hline & Trabaja por cuenta ajena & 73 & 24,7 \\
\hline & Trabaja por cuenta propia & 9 & 3 \\
\hline & Trabajo informal & 40 & 13,5 \\
\hline & Jubilado/a, Retirado/a & 1 & 0,3 \\
\hline \multirow{6}{*}{ Nivel socioeconómico aproximado cuando era un niño } & Bajo (0-19999 euros) & 35 & 11,8 \\
\hline & Medio-bajo (20 000-39 999 euros) & 74 & 25 \\
\hline & Medio (40 000-59 000 euros) & 102 & 34,5 \\
\hline & Medio-alto (60 000-79 999 euros) & 32 & 10,8 \\
\hline & Alto (+80000 euros) & 3 & 1 \\
\hline & NS/NC & 50 & 16,9 \\
\hline \multirow{6}{*}{ Nivel socioeconómico aproximado en la actualidad } & Bajo (0-19999 euros) & 55 & 18,6 \\
\hline & Medio-bajo (20 000-39 999 euros) & 61 & 20,6 \\
\hline & Medio (40 000-59 000 euros) & 107 & 36,1 \\
\hline & Medio-alto (60 000-79 999 euros) & 27 & 9,1 \\
\hline & Alto (+80000 euros) & 1 & 0,3 \\
\hline & NS/NC & 45 & 15,2 \\
\hline
\end{tabular}

Base $\mathrm{n} .=296$

\subsection{Análisis}

Para el análisis de los resultados de la escala se ha empleado el programa estadístico IBM-SPSS (versión 22). En primer lugar, se han calculado los resultados para cada subescala, haciendo la media de la suma de las puntuaciones de cada uno de los ítems relativos a cada subescala, sin ponderar, tal y como recomiendan los propios autores (Guyton y Wesche, 2005: 25). De esta forma, teniendo en cuenta que cada una se construye en base a una escala Likert 1-4, las puntuaciones de cada subescala serían las siguientes (Guyton y Wesche, 2005: 25):

- Subescala de Experiencia (7 ítems): puntuación entre 7 y 28, donde los rangos serían 1-15 (bajo), 16-24 (medio), 24-28 (muy positivo).

- Subescala de Actitud: (7 ítems) puntuación entre 7 y 28, donde los rangos serían 1-15 (bajo), 16-24 (medio), 24-28 (muy positivo).

- Subescala de Eficacia: (20 ítems) puntuación entre 20 y 80, donde los rangos serían 20-54 (bajo), 55-66 (medio), 67-80 (muy positivo).

No obstante, en el presente estudio, dado que los ítems no están ponderados, para facilitar la interpretación del análisis, hemos propuesto la construcción de un índice 1-10 para cada una de las subescalas (dividiendo por 2,8; 2,8; y 8,0 las puntuaciones anteriores respectivamente), dando lugar a tres índices sobre 10, teniendo en cuenta los rangos anteriores propuestos por los autores:

- Índice de Experiencia Intercultural (IEI): puntuación 1-10, donde los rangos serían 1-5,4 (bajo), 5,5-8,6 (medio), 8,7-10 (muy positivo).

- Índice de Actitud Intercultural (IAI): puntuación 1-10, donde los rangos serían 1-5,4 (bajo), 5,58,6 (medio), 8,7-10 (muy positivo).

- Índice de Eficacia Intercultural (IEFI): puntuación 1-10, donde los rangos serían 1-6,8 (bajo), 6,9-8,3 (medio), 8,4-10 (muy positivo). 
Especificar un índice sobre diez facilita la interpretación de los resultados, así como las comparativas entre las escalas. Para validar este índice se han replicado todos los análisis tanto en el rango propuesto por los autores en la publicación original, como el índice sobre diez que proponemos en la presente investigación, obteniéndose los mismos resultados.

Por otro lado, dado que la pregunta de «Enfoque» no se realiza mediante escala, se ha optado por hacer un simple análisis descriptivo de los resultados obtenidos tanto en general, como en relación a las categorías cualitativas de la escala.

Una vez recalculados los índices, se ha procedido a realizar un análisis descriptivo de los resultados, teniendo en cuenta la media de cada uno de ellos, y su desviación estándar (DS). A continuación, y empleando técnicas multivariantes, se ha procedido a analizar la incidencia de las características sociopersonales y educativas en cada uno de los tres índices, mediante un análisis empleando la prueba de T de Student para las variables dicotómicas (género), y un análisis de la varianza (ANOVA), con posthoc a través de la prueba de Bonferroni para el resto de variables, encontrándose algunas relaciones significativas.

Finalmente, se procedió a explorar la posible relación entre los tres índices analizados en el estudio (IEI, IAI e IEFI). En primer lugar, se calculó el coeficiente de correlación de Pearson entre las variables. Tras encontrarse una relación significativa entre IAI y el IEFI, se procedió a realizar una regresión lineal múltiple estándar, para determinar si, como expresa la literatura al respecto, la experiencia y la actitud interculturales pueden influir en una mayor eficacia en este sentido en los futuros docentes. El modelo cumplía los supuestos de normalidad. Se confirmó la correlación no muy elevada entre las variables $<, 3$, pero significativa en el caso del IAI y el IEFI. El valor de tolerancia $(0,99)$ y el VIF $(1,00)$ indicaban que no existe multicolinealidad. Tanto el gráfico de residuo estandarizado, como el diagrama de dispersión, indicaron que se cumplen las condiciones de linealidad, homocedasticidad e independencia de los residuos (siguiendo los criterios de Tabachnicky Fidell, 2001).

\section{Resultados}

En primer lugar, atendiendo al análisis descriptivo de los resultados, podemos apreciar que, en los tres índices, nuestros y nuestras participantes se sitúan en el rango medio, si bien la experiencia intercultural previa es la que obtiene una puntuación media más baja $(\mu=6,97)$, aunque con una desviación estándar superior $(\sigma=1,38)$. Tanto la actitud como la eficacia tienen una puntuación media elevada, sin llegar a alcanzar el rango de «muy positivo» $(\mu=8,06$ y $\mu=8,14$ respectivamente).

Tabla III. Valores estadísticos para los índices IEI, IAI, IEFI

\begin{tabular}{lcc}
\hline & $\mu$ & $\sigma$ \\
\hline IEI & 6,97 & 1,38 \\
\hline IAI & 8,06 & 1,03 \\
\hline IEFI & 8,14 & 1,27 \\
\hline
\end{tabular}

Sin embargo, en el caso de la pregunta destinada a conocer el enfoque de la interculturalidad adoptado por los y las estudiantes de Magisterio y del Máster de Profesorado, la mayoría se queda en el primer paso de la gestión intercultural (según Guyton y Wesche, 2005). Un 50,3\% de las personas encuestadas 
se adhiere a la denominada «visión basada en la tolerancia», la cual queda lejos de las dos visiones que se consideran dentro de un enfoque intercultural más amplio e inclusivo («visión intercultural» con un $12,5 \%$, y visión «intercultural» fundamentada en el apoyo social con un 15,9\%).

\section{Tabla IV. Distribución de la muestra por enfoque principal adoptado} frente a la educación intercultural

\begin{tabular}{lcc}
\hline & FRECUENCIA & PORCENTAJE \\
\hline a) Visión basada en la «tolerancia» & 149 & 50,3 \\
\hline b) Visión asimilacionista» & 21 & 7,1 \\
\hline c) Visión desde la «identidad singular de cada cultura» & 42 & 14,2 \\
\hline d) Visión «intercultural» & 37 & 12,5 \\
\hline e) Visión «intercultural» fundamentada en el apoyo social & 47 & 15,9 \\
\hline
\end{tabular}

A continuación, se trató de determinar si las variables sociopersonales y educativas incluidas en la escala podían determinar una mayor o menor puntuación en los tres índices analizados (IEI, IAI, IEFI), mediante las pruebas de T de Student para variables dicotómicas y ANOVA para las de respuesta múltiple. De las variables independientes analizadas, no resultaron significativas las diferencias entre religión, nivel de estudios del padre y la madre, situación laboral de la persona encuestada y nivel socioeconómico actual.

Sin embargo, las diferencias entre sexo, grupo de edad, tipo de estudios cursados y nivel socioeconómico en la infancia sí resultaron significativas entre algunos de los grupos. Así, la diferencia de las medias entre hombres y mujeres resultaban relevantes, a favor de las últimas ( 0,8 puntos más) a la hora de medir la eficacia intercultural. En cuanto al grupo de edad, no se encontraron diferencias importantes en el IEFI, pero sí en la experiencia intercultural, más elevada en el caso del grupo más joven $(\mu=7,2)$ respecto a los mayores de 32 años, y en la actitud intercultural entre los grupos de 32 a 37 y 38 a 44 años, más elevada en el caso de los primeros $(\mu=8,3)$. Nuevamente atendiendo al índice de eficacia, los estudios cursados pueden ser una variable relevante. En nuestra muestra, existía una diferencia significativa entre el alumnado de Magisterio Infantil $(\mu=8,5)$ y del Máster de Profesorado de Secundaria $(\mu=7,8)$. Por último, el nivel socioeconómico cuando el/la participante era un/a niño/a determinaba una puntuación mucho menor en experiencia intercultural para aquéllos/as que declaraban encontrarse en un nivel alto $(\mu=4,6)$, respecto a los niveles medio-alto y medio. Por otro lado, los/las que declaraban haber tenido un estatus socioeconómico medio tenían una media de puntuación de eficacia intercultural significativamente más alta que los/las participantes de nivel socioeconómico bajo (0,8 puntos más). 
Tabla V. Valoración de los índices IEI, IAI, IEFI por variables sociopersonales y educativas (Media y nivel de significación)

\begin{tabular}{|c|c|c|c|}
\hline \multirow[t]{2}{*}{ VARIABLES INDEPENDIENTES } & \multicolumn{3}{|c|}{ INDICADORES } \\
\hline & IEI & $|A|$ & IEFI \\
\hline \multicolumn{4}{|l|}{ Sexo } \\
\hline Hombre & 6,7 & 7,9 & $7,5^{\star \star}$ \\
\hline Mujer & 7 & 8,1 & $8,3^{\star \star}$ \\
\hline \multicolumn{4}{|l|}{ Grupo de edad } \\
\hline $20-25$ & $7,2^{\star}$ & 8,1 & 8,1 \\
\hline $26-31$ & 6,8 & 7,9 & 8,2 \\
\hline $32-37$ & $6,3^{*}$ & $8,3^{\star}$ & 7,8 \\
\hline $38-44$ & 6,4 & $7,2^{*}$ & 8,3 \\
\hline \multicolumn{4}{|l|}{ Estudios cursados } \\
\hline Magisterio de Infantil & 7 & 8,1 & $8,5^{\star \star}$ \\
\hline Magisterio de Primaria & 7,1 & 8,3 & 8,1 \\
\hline Máster Profesorado de Secundaria & 6,9 & 7,9 & $7,8^{\star \star}$ \\
\hline \multicolumn{4}{|l|}{ Nivel socioeconómico cuando era niño/a } \\
\hline Bajo (0-19999€) & 7 & 8,3 & $7,5^{\star}$ \\
\hline Medio- Baio (20 000-39999€) & 6,8 & 8,1 & 8,3 \\
\hline Medio (40000-59999€) & $7,0^{*}$ & 7,9 & $8,3^{*}$ \\
\hline Medio-Alto (60 000-79 999€) & $7,3^{\star}$ & 8,2 & 8,1 \\
\hline Alto (80000 euros o más) & $4,6^{\star}$ & 7,6 & 8,2 \\
\hline
\end{tabular}

$*_{\mathrm{p}}<, 05, *^{*} \mathrm{p}<, 01$.

Mediante el cálculo de correlación de Pearson entre los tres índices, se estableció una interdependencia leve $(\mathrm{r}<, 3)$ pero significativa $(\mathrm{p}<, 05)$ entre los índices de actitud y eficacia intercultural (Tabla VI).

Debido a ello, a continuación, se realizó un análisis de regresión múltiple estándar, para determinar si, como expresan trabajos previos, la experiencia y la actitud interculturales pueden influir en una mayor eficacia en este sentido en los/las futuros/as docentes. El modelo resultante explicaba el 4,5\% de la varianza del IEFI, siendo estadísticamente significativo $(\mathrm{p}=, 001)$. La variable que influía de manera significativa en la eficacia era la actitud (beta $=, 202 ; \mathrm{p}=, 001)$. (Ver Tabla VII).

Tabla VI. Correlación entre los índices IEI, IAI, IEFI

\begin{tabular}{lccc}
\hline & IEI & IAI & IEFI \\
\hline IEI & 1 &, 061 &, 085 \\
\hline IAI &, 061 & 1 &, $145^{\star}$ \\
\hline IEFI &, 085 &, $145^{\star}$ & 1 \\
\hline
\end{tabular}

$*_{\mathrm{p}}<, 05$. 
Tabla VII. Modelo de regresión lineal múltiple IEFI

\begin{tabular}{|c|c|c|c|c|c|c|}
\hline & & \multicolumn{2}{|c|}{ Coeficientes no estandarizados } & \multicolumn{3}{|c|}{ Coeficientes estandarizados } \\
\hline \multicolumn{2}{|c|}{ Modelo } & B & Error estándar & Beta & $t$ & Sig. \\
\hline \multirow[t]{3}{*}{1} & (Constante) & 6,088 &, 599 & & 10,158 &, 000 \\
\hline & $|A|$ & ,224 &, 064 &, 202 & 3,500 &, 001 \\
\hline & IE &, 045 & 048 &, 055 &, 949 & ,343 \\
\hline
\end{tabular}

Variable dependiente: IEFI.

\section{Discusión}

Como se ha expresado en la introducción, el propósito de esta investigación ha sido doble. Por un lado, metodológica, ya que se ha adaptado una herramienta internacional (MES, de Guyton y Wesche, 2005) al idioma y contexto españoles, dando lugar a la EEI, testando su fiabilidad con técnicas estadísticas, y su validez en la presente discusión de resultados. Por otra parte, ha tenido un fin exploratorio para conocer el nivel de eficacia intercultural que se auto atribuye el profesorado de Infantil, Primaria y Secundaria en formación en España, a través de esta herramienta.

En cuanto al primer objetivo, a pesar de que hemos propuesto una nueva interpretación de resultados a través de índices 1-10 (IEI, IAI, IEFI) para facilitar su comprensión, las puntuaciones obtenidas están en la línea de investigaciones a nivel internacional que han empleado la MES. Los propios Guyton y Wesche (2005) especificaban en el estudio que testaba su escala que la mayoría del profesorado puntuaba «alto» en actitud intercultural y «medio-alto» en eficacia intercultural. En nuestro caso, en ambos índices, IAI e IEFI, la puntuación media estaría en el rango de medio-alto (8,06 y 8,14 respectivamente, sobre 10). En la misma línea de resultados se encontraban otros estudios que habían empleado la MES (Nadelson, 2012; Silverman, 2015; Seak-Zoon, 2015; Mansuri, 2017). Así, tanto el test realizado a través del cálculo del alpha de Cronbach, como la comparativa de los resultados con otros estudios previos, validarían la EEI como instrumento de medida de la eficacia intercultural del profesorado y del profesorado en formación. Su estructura interna también sería consistente si tenemos en cuenta el análisis factorial realizado.

En segundo lugar, en cuanto a los resultados del EEI como herramienta exploratoria para analizar esta eficacia intercultural en España, el hecho de que las puntuaciones en los índices de actitud y eficacia se encuentren casi al nivel de la marca «muy positivo», alinean este estudio con otros previos realizados en nuestro país, pese a que éstos utilizaron otras herramientas de investigación. Aguaded, De la Rubia y González (2013) encontraron que el profesorado de todas las etapas educativas, consideraba que es eficaz interculturalmente y cree utilizar técnicas para aplicar la educación intercultural. Sin embargo, a la hora de abordar un enfoque concreto en el aula para tratarlo, los y las participantes no manifestaban un conocimiento real. Tal y como también se desprendía del trabajo de Díez Gutiérrez (2014) con profesorado de Secundaria, o Gil Madrona, Gómez-Barretoy González-Víllora (2016) con profesorado en formación. De manera similar, en los resultados de nuestro estudio, al preguntar precisamente por cómo enfocar esta interculturalidad en el aula, la mayoría (50,3\%) se limitaron a la visión de la «tolerancia» de las diferentes culturas. Conclusión similar a la que llegaron en el estudio con profesorado en formación, es decir, la ausencia de un conocimiento real sobre qué es la educación intercultural en el aula.

En cuanto a la influencia de las variables sociopersonales, trabajos como el de Nadelson (2012) indicaban que no era significativa. Sin embargo, en nuestro estudio, el hecho de ser mujer y partir de un nivel 
familiar socioeconómico medio influían de manera relevante en un mayor IEFI, así como el ser joven (20-25) en el IEI, o estudiar de manera más tardía (32-37 años) en un mayor IAI. La formación recibida también resulta una variable importante en la eficacia intercultural, ya que el alumnado de Magisterio de Infantil tenía un índice significativamente mayor que el del Máster de Profesorado de Secundaria. Ello puede deberse a que en este grado el alumnado tiene asignaturas específicas sobre interculturalidad y diversidad, y es un tema que se trata de manera tanto directa en la mayoría de universidades españolas (en la Universidad de Zaragoza, de donde procede una gran parte de la muestra, se imparte en la asignatura «Educación Intercultural») y transversal durante 4 años, a diferencia del alumnado del Máster, que no tienen asignaturas específicas sobre el tema. Como ya se ha mencionado, diversos estudios a nivel internacional (Guyton y Wesche, 2005; Mitchell, 2009) y nacional (Aguado Odina, Gil Jaurena y Mata Benito, 2008; Goenechea, 2008; Jordán, 2007) remarcan la formación como principal herramienta para poseer una mayor eficacia intercultural en el aula.

Otra de las cuestiones que pretendíamos evidenciar mediante la EEI es si la experiencia o la actitud interculturales influían en una mayor eficacia en este sentido. En nuestro estudio, el IEI no parece ser decisivo, como propugnaban Silverman (2015) o Seak-Zoon (2015), si bien el IAI sí influía significativamente en el IEFI, lo que ayudaría a defender la idea de que cualquier estudiante, independientemente de su experiencia previa con personas diversas, puede alcanzar un nivel alto de eficacia si tiene una actitud proclive a ello. Lo cual nos lleva de nuevo al tema de la importancia de la formación en este sentido como medio para lograr profesorado interculturalmente eficaz, ausente de prejuicios y que, consecuentemente, logre que su alumnado diverso tenga niveles de rendimiento similares al alumnado nativo, tal y como propugnaban Martin y Dagostino-Kalniz (2015), Gómez Barreto, Gil Madrona y Martínez (2017), Atwater, Lance, Woodard y Johnson (2013), Im y Martin (2015) o Kirch, Bargerhuff, Cowan y Wheatly, 2007).

\section{Conclusiones}

A pesar de la presencia relevante de alumnado inmigrante en las aulas y las diferencias en sus resultados académicos con el alumnado nativo, las prácticas docentes todavía no son eficazmente interculturales. Prueba de ello son los resultados del presente estudio exploratorio y sus precedentes, donde, a pesar de la autoconsideración eficaz del profesorado en formación, sus enfoques de cómo plasmar la educación intercultural en las aulas se queda en la superficie.

Es por ello que las iniciativas de mensurar la eficacia de los y las docentes en activo y aquéllos y aquéllas que se encuentran en formación adquieren un sentido relevante. De esta manera, podemos identificar esta cuestión, para, así, proponer medidas (fundamentalmente basadas en incrementar la formación en educación intercultural de este colectivo) que favorezcan aulas inclusivas y la transformación social. Para contribuir a ello, el presente estudio muestra una propuesta de medición adaptada del mundo anglosajón a través de la EEI, así como un análisis exploratorio del profesorado en formación de todas las etapas de escolarización. Éste puede servir de primer acercamiento a una situación que merece ser profundizada con muestras más amplias, o con otras herramientas de tipo cualitativo.

Sólo a través de un profesorado interculturalmente eficaz e intelectualmente transformador puede lograr superarse la exclusión social que supone que el alumnado diverso se vaya quedando atrás, abogando por la igualdad de las diferencias. 


\section{Referencias bibliográficas}

Aguaded, Eva Má ; De la Rubia, Pablo y González, Esther (2013): "La importancia de la formación del profesorado en competencias interculturales". Profesorado: Revista de curriculum y formación del profesorado, $17(1), 339-365$.

Aguado Odina, Ma Teresa; Gil Jaurena, Inés y Mata Benito, Patricia (2008): "El enfoque intercultural en la formación del profesorado. Dilemas y propuestas". Revista Complutense de Educación, 19 (2), 275-292.

Anders, T.D.; Martin, C.E. y Yarbrough, D. (1990). Social Diversity Survey (SDS): Research edition. Cumming, GA: Office of Disability Services.

Atwater, Mary; Lance, Jennifer; Woodard. UrLeaka y Johnson, Natasha H. (2013): "Race and Ethnicity: Powerful Cultural Forecasters of Science Learning and Performance". Theory Into Practice, 52 (1), 6-13. DOI: https://doi.org/10.1080/07351690.2013.743757.

Bennett, Christine; Niggle, Timothy y Stage, Frances (1990): "Preservice multicultural teacher education: Predictors of student readiness". Teaching and Teacher Education, 6, 243-254.

Broc, Miguel (2018): "Academic Performance and Other Psychological, Social and Family Factors in Compulsory Secondary Education Students in a Multicultural Context". International Journal of Sociology of Education, 7 (1), 1-23. DOI: https://doi.org/10.17583/rise.2018.2846.

Campbell, R. L. y Farrell, R. V. (1985): “The Identification of Competencies for Multicultural Teacher Education”. The Negro Education Review, 36, 137-144.

Carrillo, Anna; Girbés-Peco, Sandra; De Botton, Lena y Valls-Carol, Rosa (2017): “The Role of Communicative Acts in the Dream Process: Engaging Moroccan Migrants in a Community Development Initiative in Urban Spain”. Community Development Journal, 1-18. DOI: https://doi.org/10.1093/cdj/bsx049.

Cooper, A., Beare, P., y Thorman, J. (1990): "Preparing Teachers for Diversity: A Comparison of Student Teaching Experiences in Minnesota and South Texas". Action in Teacher Education, 12, 23-30.

Cummins, Jim (2001): “Empowering Minority Students: A framework for Intervention”. Harvard Educational Review, 71 (4), 18-37.

Díez Gutiérrez, Enrique Javier (2014): “La práctica educativa intercultural en secundaria”. Revista de Educación, 363, Enero-Abril, 12-34.

Espinosa, Anabel (2014): Teachers' Educational Beliefs and Culturally and Linguistically Diverse Students within Response to Intervention. Miami: University of Miami.

Flecha, Ramón; y Soler, Marta (2013): “Turning Difficulties into Possibilities: Engaging Roma Families and Students in School Through Dialogic Learning”. Cambridge Journal of Education, 43 (4), 451-465. DOI: https://doi.org/10.1080/0305764X.2013.819068.

Gao, Wei y Mager, Gerald (2011): “Enhancing Preservice Teachers' Sense of Efficacy and Attitudes Toward School Diversity Through Preparation: A case of one U.S. Inclusive Teacher Education Program". International Journal of Special Education, 26, 89-104. 
Gil-Madrona, Pedro; Gómez-Barreto, Isabel y González-Víllora, Sixto (2016): “Percepción de los estudiantes de maestro de educación infantil sobre su formación intercultural”. Magis: Revista Internacional de Investigación en Educación, 9 (18), 111-128. DOI: https://doi.org/10.11144/Javeriana.m9-18.pmei.

Goenechea, Cristina (2008): “¿Es la formación del profesorado la clave de la educación intercultural?”. Revista Española de Pedagogía, 239, 119-136.

Gómez Barreto, Isabel; Gil-Madrona, Pedro y Martínez, María (2017): "Valoración de la competencia intercultural en la formación inicial de los maestros de Educación Infantil”. Interciencia, 42 (8), 484-493.

García-Carrión, Rocío; Molina-Luque, Fidel y Roldán, Silvia (2017): “How Do Vulnerable Youth Complete Secondary Education? The Key Role of Families and the Community". Journal of Youth Studies, 27 (14), 1-16. DOI: https://doi.org/10.1080/13676261.2017.1406660.

Guyton, E.M., Wesche, M. V. (2012): “Multicultural Efficacy Scale. Development, Item Selection, and Reliability”. Multicultural Perspectives, 7 (4), 21-29.

Herrera, Azucena Leticia (2018): "Decálogo de competencias y sus indicadores para gestión de capital humano universitario". Multidisciplinary Journal of Educational Research, 8(1), 56-85. DOI: https://doi. org/10.17583/remie.2018.2990.

Howard, T. C. y Aleman, G. R. (2008): “Teacher Capacity for Diverse Learners; What Do Teachers Need to Know?" en Cochran-Smith, M.; Feiman-Nemser, S. et al., (eds.): Handbook of Research on Teacher Education: Enduring Questions in Changing Contexts. New York: Macmillan.

Im, Sungmin y Martin, Sonya (2015): "Using Cogenerative Dialogues to Improve Coteaching for Language Learner (LL) Students in an Inclusion Science Classroom”. Asia-Pacific Journal of Teacher Education, $43(4), 355-369$.

Jordán, Josep A. (2007): “Formación intercultural del profesorado de secundaria”. Estudios sobre Educación, $12,59-80$.

Kirch, S. A.; Bargerhuff, M.E.; Cowan, H. y Wheatly, M. (2007): "Reflections of Educators in Pursuit of Inclusive Science Classrooms”. Journal of Science Teacher Education, 18 (4), 663-692.

Larke, Patricia J. (1990): “Cultural Diversity Awareness Inventory: Assessing the Sensitivity of Preservice Teachers". Action in Teacher Education, 12, 23-30.

Mansuri, L.J. (2017): "Multicultural Efficacy of Preservice Teachers: A Study". Scholarly Research Journal for Humanity Science y English Language, 4(23).

Ministerio de Educación, Cultura y Deporte (2018): Estadísticas de enseñanzas no universitarias. Recuperado de https://www.mecd.gob.es/servicios-al-ciudadano-mecd/estadisticas/educacion/no-universitaria. html.

Mitchell, Laura A. (2009). Becoming Culturally Responsive Teachers in Today's Diverse Classroom. American Educational Research Association Meeting: Annual, San Diego. 
Nadelson, L.S.; Boham, M. D.; Conlon-Khan, L.; Fuentealba, M.J.; Hall, C.J.; Hoetker, G. A. y Zenkert, A.J. (2012): “A Shifting Paradigm: Preservice Teachers' Multicultural Attitudes and Efficacy". Urban Education, 47 (6), 1183-1208.

OCDE (2016a): Programme for International Student Assessment: PIS A 2015 Edition. Recuperado de http:// www.oecd.org/pisa/.

OCDE (2016b): Global Competency for an Inclusive World. Recuperado de https://www.oecd.org/education/ Global-competency-for-an-inclusive-world.pdf.

Ohio State University. (1988): Results From a Survey of Multicultural Attitudes and Competencies Among Students Sompleting Student Teaching From the College of Education at the Obio State University, 1985-1986. Columbus, OH. (ERIC Document Reproduction Service No. ED293793).

Parekh, Bhikhu (2006): Rethinking Multiculturalism: Cultural Diversity and Political Theory. Basingstoke: Palgrave Macmillan.

Park, Jennifer; Chu, Hye-Eun y Martin, Sonya N. (2016): “Exploring How Korean Teacher's Attitudes and Self-Efficacy for Using Inquiry and Language-Based Teaching Practices Impacts Learning for Culturally and Linguistically Diverse Students: Implications for Science Teacher Education”. Eurasia Journal of Mathematics, Science y Technology Education, 12 (7), 1799-1841. DOI: https://doi.org/10.12973/eurasia.2016.1536a.

Romero, Carlos Giménez (2003): "Pluralismo, multiculturalismo e interculturalidad”. Educación y Futuro: revista de investigación aplicada y experiencias educativas, 8, 11-20.

Silverman, S. K. (2008): “The Teachers' Sense of Multicultural Efficacy Scale: Construction and Validation". F. Hayes Graduate Research Forum, 22 $2^{\text {nd }}$.

Seak-Zoon, Roh (2015): "Exploring the Multicultural Efficacy of Korean Pre-Service Secondary School Teachers and their Experience toward Multiculturalism and Multicultural Education”. International Journal Of U-AndE-Service, Science And Technology, 8, 179. DOI: https://doi.org/10.14257/ijunesst.2015.8.8.18.

Sleeter, Christine E. y Grant, Carl A. (1987): “The Analysis of Multicultural Education in the United States". Harvard Educational Review, 57 (4), 421-444.

Tabachnick, Barbara G. y Fidell, L. S. (2001). Using Multivariate Statistics (4 $4^{\text {th }}$ edn). NewYork: HarperCollins.

\section{Notas biográficas}

Tatiana Iñiguez-Berrozpe es profesora Ayudante Doctor en el área de Sociología del Departamento de Psicología y Sociología de la Universidad de Zaragoza (Facultad de Educación). Pertenece al Grupo de Investigación EDUCAVIVA (Gobierno de Aragón). También es editora adjunta de la Revista Internacional de Sociología de la Educación (RISE) y editora consultiva de Adult Education Quarterly (AEQ). Su principal área de investigación es la Sociología de la Educación (Educación de Personas Adultas, Aprendizaje Permanente, Educación Inclusiva y Convivencia Escolar). 
Diana Valero es profesora Ayudante Doctor en el área de Trabajo Social del Departamento de Psicología y Sociología de la Universidad de Zaragoza (Facultad de Ciencias Sociales y del Trabajo). Su principal área de investigación es la inclusión social y educativa, especialmente de colectivos vulnerables (inmigrantes, Roma, adultos mayores). Ha participado en diversos proyectos nacionales e internacionales en este ámbito y publicado en revistas de referencia como International Journal of Inclusive Education.

Ainhoa Flecha es profesora de Sociología en la Universidad Autónoma de Barcelona. Sus intereses de investigación incluyen grupos culturales, género y sociología de la educación. En estas líneas ha desarrollado varios proyectos nacionales y europeos, y publicado en revistas de referencia en el área.

Gisela Redondo-Sama es doctora en Sociología y ha sido Marie Curie Postdoctoral Fellow en la Universidad de Cambridge. Actualmente es investigadora Ramón y Cajal en el Centro de Ética Aplicada de la Universidad de Deusto donde desarrolla la línea de investigación de liderazgo dialógico. 